\section{Dissipativity and stability of a class of nonlinear multiple time delay integro-differential equations}

\author{
Chaolong Zhang ${ }^{a}$, Feiqi Deng ${ }^{\mathrm{b}, *}$, Haoyi Moc${ }^{c}$, Hongwei Ren ${ }^{d}$ \\ ${ }^{a}$ College of Computational Science, Zhongkai University of Agriculture and Engineering, Guangzhou, 510225, P. R. China. \\ ${ }^{b}$ Systems Engineering Institute, South China University of Technology, Guangzhou, 510640, P. R. China. \\ ${ }^{c}$ School of Applied Mathematics, Guangdong University of Technology, Guangzhou, 510006, P. R. China. \\ ${ }^{d}$ School of computer and electronic information, Guangdong University of Petrochemical Technology, Maoming 525000, P. R. China.
}

\begin{abstract}
This paper is concerned with the dissipativity and stability of the theoretical solutions of a class of nonlinear multiple time delay integro-differential equations. At the first, we give a generalized Halanay inequality which plays an important role in the study of dissipativity and stability of integro-differential equations. Then, we apply the generalized Halanay inequality to the dissipativity and the stability the theoretical solution of delay integro-differential equations (or by small $\epsilon$ perturbed) and some interesting results are obtained. Our results generalize a few previous known results. Finally, two examples are provided to demonstrated the effectiveness and advantage of the theoretical results.
\end{abstract}

Keywords: Delay integro-differential equations, dynamical systems, Halanay inequality, dissipativity, stability.

2010 MSC: 93Dxx, 45Gxx, 47H14.

(C)2019 All rights reserved.

\section{Introduction}

In the recent 40 years, the theories for delay differential equations (DDEs), delay integro- differential equations (DIDEs) and neutral delay differential equations (NDDEs) have been studied by many authors and a great deal of interesting results have been obtained.

In the study of dynamical systems, it is often the asymptotic behavior of the system that is of interest, and it is highly desirable to the dissipativity of the underlying system. The concept of global dissipativity as pointed out in [13] is a more general concept in dynamical systems and it has found applications in the areas such as stability theory, chaos and synchronization theory, and robust control. Up to now, some researchers have paid particular attention to the global dissipativity of several classes of simple neural networks with delays $[1,13,19]$. Very recently, some sufficient conditions for the global dissipativity of

\footnotetext{
*Corresponding author

Email addresses: handsomezcl@126.com (Chaolong Zhang), aufqdeng@scut.edu.cn (Feiqi Deng), mhy04@163.com (Haoyi Mo), rhw-6621@163.com (Hongwei Ren)
}

doi: $10.22436 /$ jnsa.012.06.03

Received: 2017-07-05 Revised: 2018-11-23 Accepted: 2018-12-20 
a class of BAM neural networks with variable and bounded or unbounded time delays are derived by means of the Lyapunov functions and linear matrix inequality techniques [27].

However, the study on the dissipativity and stability of the integral differential equations is not much. In fact, delay integro-differential systems is widely existed.

For some examples:

(i) Volterra delay integro-differential system,

$$
\left\{\begin{array}{lc}
x^{\prime}(t)=g\left(t, x(t), x(t-\eta(t)), \int_{0}^{\sigma(t)} K(t, s, x(t-s)) d s\right), & t \geqslant 0, \\
x(t)=\phi(t), & -\infty<t \leqslant 0 ;
\end{array}\right.
$$

(ii) following integro-differential neural network system

$$
\frac{d x_{i}(t)}{d t}=-d_{i}(t) x_{i}(t)+\sum_{j=1}^{n} a_{i j}(t) f_{j}\left(x_{j}(t)\right)+\sum_{j=1}^{n} \int_{0}^{\infty} K_{i j}(t, s) g_{j}\left(x_{j}(t-s)\right) d s+I_{i}(t),
$$

where $i=1,2, \ldots, n$;

(iii) perturbed delay integro-differential equations by small $\epsilon$,

$$
\begin{cases}\epsilon y^{\prime}(t, \epsilon)=f\left(t, y(t, \epsilon), y(t-\tau(t), \epsilon), \int_{0}^{\sigma(t)} K(s) y((t-s), \epsilon) d s\right), & t \geqslant t_{0}, \\ y(t, \epsilon)=\phi(s), & t \leqslant t_{0} .\end{cases}
$$

From the (1.1)-(1.3), we all know that those nonlinear integro-differential systems have the time-varying delays and distributed delays.

With the development of the theory on nonlinear differential equations (e.g., see [5, 22]), it is beneficial to obtain some new nonlinear general delay differential inequalities. It is well known that inequalities such as the Halanay inequality [6], are important methods for investigating the dynamical behavior of differential equations.

Halanay's inequality: If $a>b>0, \tau>0$ and

$$
v^{\prime}(t) \leqslant-a v(t)+b \sup _{t-\tau \leqslant s \leqslant t} v(s), \quad t \geqslant t_{0}
$$

then there exist $\beta$ and $\alpha>0$ such that

$$
v(t) \leqslant \beta e^{-\alpha\left(t-t_{0}\right)}, \quad t \geqslant t_{0} .
$$

In recent years, various inequalities have been established such as the impulsive inequalities or the delay inequalities in $[2,3,7,8,15,16,18,20,25,28,31,32,34-36]$.

Up to now, only a few papers considered the global dissipativity of integro-differential equations with both time-varying delays and infinitely distributed time delays. In order to extend and improve the results obtained in $[3,4,8,14,17,29-31]$, this paper aims to obtain some sufficient conditions which ensuring the global dissipativity of integro-differential equations with both variable and infinitely distributed time delays. Especially, we develop a generalized Halanay inequality to approach the sufficient condition for the global dissipativity of integro-differential equations with both variable and infinitely distributed time delays, under the activation functions without satisfying the growth conditions. Furthermore, motivated by the analysis in $[12,14,23,24,26,29-31,37,38]$, global exponential dissipativity, the positive invariant sets, and globally exponential attractive sets of integro-differential equations with both variable and infinitely distributed time delays are also investigated by employing Lyapunov functions and a generalized Halanay inequality. Finally, two examples are presented to show that these dissipativity conditions are very easy-to-test by using Matlab program and Matlab LMI tool box.

The rest of the paper is organized as follows. In Section 2, we introduce a generalized Halanay inequality with multiple variable time delay. In Section 3, by using a generalized Halanay inequality, some novel sufficient conditions for dissipativity and global exponential stability of some integro-differential systems with time-varying delay is derived. In Section 4, two examples are illustrated to demonstrate the effectiveness of the obtained results. 


\section{The generalized Halanay inequality}

Theorem 2.1 (Generalized Halanay inequality). Consider the following generalized inequalities

$$
\begin{cases}v^{\prime}(t) \leqslant \gamma(t)+a(t) v(t)+\sum_{i=1}^{m} b_{i}(t)[v(t)]_{\tau_{i}(t)}+\sum_{j=1}^{n} c_{j}(t) \int_{0}^{+\infty} K_{j}(s) v(t-s) d s, & t>t_{0}, \\ v(t)=\phi(t), & t \in\left(-\infty, t_{0}\right],\end{cases}
$$

where $a(t)<0, \gamma(t) \geqslant 0, b_{i}(t) \geqslant 0, c_{j}(t) \geqslant 0, \tau_{\mathfrak{i}}(t) \geqslant 0, i=1,2, \ldots, m, j=1,2, \ldots, n, v(t) \geqslant 0,[v(t)]_{\tau_{\mathfrak{i}}(t)}=$ $\sup _{\mathrm{t}-\tau_{\mathfrak{i}}(\mathrm{t}) \leqslant s \leqslant \mathrm{t}} v(\mathrm{~s}), \phi(\mathrm{t})$ is bounded and continuous on $\left(-\infty, \mathrm{t}_{0}\right], \gamma^{*}=\sup _{\mathrm{t}_{0} \leqslant \mathrm{t}<+\infty} \gamma(\mathrm{t}), \mathrm{G}=\sup _{-\infty<\mathrm{s} \leqslant \mathrm{t}_{0}}\left|\phi(\mathrm{s})-\frac{\gamma^{*}}{\sigma}\right|$, the delay kernel $\mathrm{K}_{\mathrm{j}}(\cdot) \geqslant 0$ and $\int_{0}^{+\infty} \mathrm{K}_{\mathbf{j}}(\mathrm{s}) \mathrm{e}^{\mu \mathrm{s}} \mathrm{ds}<+\infty$ for some positive number $\mu$.

If there exists $\sigma>0$ such that

$$
a(t)+\sum_{i=1}^{m} b_{i}(t)+\sum_{j=1}^{n}\left(c_{j}(t) \int_{0}^{+\infty} k_{j}(s) d s\right) \leqslant-\sigma<0, \quad t \geqslant t_{0}
$$

then there exists a constant $\mu^{*}>0$ such that

$$
v(t) \leqslant \frac{\gamma^{*}}{\sigma}+G e^{-\mu^{*}\left(t-t_{0}\right)}, \quad t \geqslant t_{0}
$$

where $\mu^{*} \geqslant 0$ is define as

$$
\mu^{*}=\inf _{t \geqslant t_{0}}\left\{\mu+a(t)+\sum_{i=1}^{m} b_{i}(t) e^{\mu \tau_{i}(t)}+\sum_{j=1}^{n} c_{j}(t) \int_{0}^{+\infty} K_{j}(s) e^{\mu s} d s=0\right\} .
$$

Proof. We define the function $F(t, \mu)$ by

$$
F(t, \mu)=\mu+a(t)+\sum_{i=1}^{m} b_{i}(t) e^{\mu \tau_{i}(t)}+\sum_{j=1}^{n} c_{j}(t) \int_{0}^{+\infty} K_{j}(s) e^{\mu s} d s,
$$

for any given fixed $t \geqslant t_{0}$, we can obtain that

$$
F(t, 0)=a(t)+\sum_{i=1}^{m} b_{i}(t)+\sum_{j=1}^{n} c_{j}(t) \int_{0}^{+\infty} K_{j}(s) d s \leqslant-\sigma<0, \quad \lim _{\mu \rightarrow+\infty} F(t, \mu)=+\infty,
$$

and

$$
F^{\prime}(t, \mu)=1+\sum_{i=1}^{m} b_{i}(t) \tau_{i}(t) e^{\mu \tau_{i}(t)}+\sum_{j=1}^{n} c_{j}(t) \int_{0}^{+\infty} s K_{j}(s) e^{\mu s} d s>0 .
$$

Therefore, for any given $t \geqslant t_{0}$, there is a unique positive $\mu$ such that

$$
\mu+a(t)+\sum_{i=1}^{m} b_{i}(t) e^{\mu \tau_{i}(t)}+\sum_{j=1}^{n} c_{j}(t) \int_{0}^{+\infty} K_{j}(s) e^{\mu s} d s=0 .
$$

From the above equality, we obtain an implicit function $\mu(t)$ for $t \geqslant t_{0}$. By that definition, one has $\mu^{*} \geqslant 0$. Define

$$
u(t)=\left\{\begin{array}{lc}
\left(v(t)-\frac{\gamma^{*}}{\sigma}\right) e^{\mu^{*}\left(t-t_{0}\right)}, & t>t_{0}, \\
v(t)-\frac{\gamma^{*}}{\sigma}, & -\infty<t \leqslant t_{0}
\end{array}\right.
$$


from above Eq. (2.2), we have, for $t \geqslant t_{0}$,

$$
\begin{aligned}
\frac{d u(t)}{d t}= & \frac{d v(t)}{d t} e^{\mu^{*}\left(t-t_{0}\right)}+\left(v(t)-\frac{\gamma^{*}}{\sigma}\right) \mu^{*} e^{\mu^{*}\left(t-t_{0}\right)} \\
\leqslant & {\left[\gamma(t)+a(t) v(t)+\sum_{i=1}^{m} b_{i}(t)[v(t)]_{\tau_{i}}+\sum_{j=1}^{n}\left(c_{j}(t) \int_{0}^{+\infty} K_{j}(s) v(t-s) d s\right)\right] e^{\mu^{*}\left(t-t_{0}\right)} } \\
& +\left(v(t)-\frac{\gamma^{*}}{\sigma}\right) \mu^{*} e^{\mu^{*}\left(t-t_{0}\right)} \\
\leqslant & \gamma(t) e^{\mu^{*}\left(t-t_{0}\right)}+a(t) v(t) e^{\mu^{*}\left(t-t_{0}\right)}+\sum_{i=1}^{m} b_{i}(t)[v(t)]_{\tau_{i}} e^{\mu^{*}\left(t-t_{0}\right)} \\
& +\sum_{j=1}^{n}\left(c_{j}(t) \int_{0}^{+\infty} K_{j}(s) v(t-s) d s\right) e^{\mu^{*}\left(t-t_{0}\right)}+v(t) \mu^{*} e^{\mu^{*}\left(t-t_{0}\right)}-\frac{\gamma^{*}}{\sigma} \mu^{*} e^{\mu^{*}\left(t-t_{0}\right)} \\
= & \gamma(t) e^{\mu^{*}\left(t-t_{0}\right)}-\frac{\gamma^{*}}{\sigma} \mu^{*} e^{\mu^{*}\left(t-t_{0}\right)}+\left(a(t)+\mu^{*}\right) v(t) e^{\mu^{*}\left(t-t_{0}\right)} \\
& +\sum_{j=1}^{n}\left(c_{j}(t) \int_{0}^{+\infty} K_{j}(s) v(t-s) e^{\mu^{*}\left(t-s-t_{0}\right)} e^{\mu^{*} s} d s\right)+\sum_{i=1}^{m} b_{i}(t)[v(t)] \tau_{i} e^{\mu^{*}\left(t-t_{0}\right)} \\
= & \gamma(t) e^{\mu^{*}\left(t-t_{0}\right)}-\frac{\gamma^{*}}{\sigma} \mu^{*} e^{\mu^{*}\left(t-t_{0}\right)}+\left(a(t)+\mu^{*}\right) \frac{\gamma^{*}}{\sigma} e^{\mu^{*}\left(t-t_{0}\right)} \\
& +\left(a(t)+\mu^{*}\right) u(t)+\sum_{i=1}^{m} b_{i}(t)[u(t)]_{\tau_{i}}+\sum_{j=1}^{n}\left(c_{j}(t) \int_{0}^{+\infty} K_{j}(s) u(t-s) e^{\mu^{*} s} d s\right) \\
& +\sum_{i=1}^{m} b_{i}(t) e^{\mu^{*} \tau(t)}\left[u(t) \tau_{i}+\sum_{j=1}^{n}\left(c_{j}(t) \int_{0}^{+\infty} K_{j}(s) e^{\mu^{*} s} d s\right)[u(t)]_{\infty} .\right. \\
& +\sum_{j=1}^{m}\left(c_{j}(t) \int_{0}^{+\infty} K_{j}(s) \frac{\gamma^{*}}{\sigma} e^{\mu^{*}\left(t-t_{0}\right)} d s\right)+\sum_{i=1}^{m} b_{i}(t) \frac{\gamma^{*}}{\sigma} e^{\mu^{*}\left(t-t_{0}\right)} \\
& \gamma(t) e^{\mu^{*}\left(t-t_{0}\right)}+a(t) \frac{\gamma^{*}}{\sigma} e^{\mu^{*}\left(t-t_{0}\right)}+\sum_{i=1}^{m} b_{i}(t) \frac{\gamma^{*}}{\sigma} e^{\mu^{*}\left(t-t_{0}\right)} \\
& +\sum_{j=1}^{n}\left(c_{j}(t) \int_{j}(s) d s\right) \frac{\gamma^{*}}{\sigma} e^{\mu^{*}\left(t-t_{0}\right)}+\left(a(t)+\mu^{*}\right) u(t) \\
& (t) \\
& \\
&
\end{aligned}
$$

Since $v(t)$ is continuous and defined for $t \in\left(-\infty, t_{0}\right]$, we let

$$
\sup _{\mathrm{t}_{0}-\tau\left(\mathrm{t}_{0}\right) \leqslant \mathrm{t} \leqslant \mathrm{t}_{0}}\left|v(\mathrm{t})-\frac{\gamma^{*}}{\sigma}\right|=\mathrm{G} .
$$

Let $\delta>1$ be arbitrary, we have $u(t)<\delta G$ for $t \in\left(-\infty, t_{0}\right]$. We claim

$$
u(t)<\delta G, \quad t>t_{0} .
$$

Suppose $u(t)<\delta G$ does not hold for $t>t_{0}$. Let

$$
t_{1}=\inf \left\{t \mid u(t)=\delta G, \quad t>t_{0}\right\},
$$

then

$$
\left\{\begin{array}{l}
u(t)<\delta G, \quad-\infty<t<t_{1} \\
u\left(t_{1}\right)=\delta G
\end{array}\right.
$$


and

$$
\left.\frac{\mathrm{d} u(\mathrm{t})}{\mathrm{dt}}\right|_{\mathrm{t}=\mathrm{t}_{1}} \geqslant 0
$$

From (2.3) and (2.4), it yields

$$
\begin{aligned}
0 \leqslant\left.\frac{d u(t)}{d t}\right|_{t=t_{1}} \leqslant & \gamma\left(t_{1}\right) e^{\mu^{*}\left(t_{1}-t_{0}\right)}+\left(a\left(t_{1}\right)+\sum_{i=1}^{m} b_{i}\left(t_{1}\right)+\sum_{j=1}^{n}\left(c_{j}\left(t_{1}\right) \int_{0}^{+\infty} K_{j}(s) d s\right)\right) \frac{\gamma^{*}}{\sigma} e^{\mu^{*}\left(t_{1}-t_{0}\right)} \\
& +\left(\mu^{*}+a\left(t_{1}\right)+\sum_{i=1}^{m} b_{i}\left(t_{1}\right) e^{\mu^{*} \tau\left(t_{1}\right)}+\sum_{j=1}^{n}\left(c_{j}\left(t_{1}\right) \int_{0}^{+\infty} K_{j}(s) e^{\mu^{*} s} d s\right)\right) \delta G \\
\leqslant & \gamma^{*} e^{\mu^{*}\left(t_{1}-t_{0}\right)}\left(1+\frac{a\left(t_{1}\right)+\sum_{i=1}^{m} b_{i}\left(t_{1}\right)+\sum_{j=1}^{n}\left(c_{j}\left(t_{1}\right) \int_{0}^{+\infty} K_{j}(s) d s\right)}{\sigma}\right)<0
\end{aligned}
$$

which contradicts (2.4). So $u(t)<\delta G$ for $t>t_{0}$. Let $\delta \rightarrow 1, u \leqslant G$, so, we have $v(t) \leqslant \frac{\gamma^{*}}{\sigma}+G e^{-\mu^{*}\left(t-t_{0}\right)}$. So, the proof of the Theorem 2.1 is completed.

Remark 2.2. If $\gamma(t) \equiv 0, c_{j}(t) \equiv 0(j=1,2, \ldots, n)$, then the inequality (2.1) can be rewritten as

$$
\left\{\begin{array}{l}
v^{\prime}(t) \leqslant a(t) v(t)+\sum_{i=1}^{m} b_{i}(t)[v(t)]_{\tau_{i}} \\
v(t)=\phi(t), \quad t \in\left(-\infty, t_{0}\right] .
\end{array}\right.
$$

If $\gamma(t) \equiv 0, b_{i}(t) \equiv 0(i=1,2 \ldots, m)$, then the inequality (2.1) can be rewritten as

$$
\left\{\begin{array}{l}
v^{\prime}(t) \leqslant a(t) v(t)+\sum_{j=1}^{n} c_{j}(t) \int_{0}^{+\infty} K_{j}(s) v(t-s) d s \\
v(t)=\phi(t), \quad t \in\left(-\infty, t_{0}\right] .
\end{array}\right.
$$

If $c_{j}(t) \equiv 0(j=1,2, \ldots, n)$, then the inequality (2.1) can be rewritten as

$$
\left\{\begin{array}{l}
v^{\prime}(t) \leqslant \gamma(t)+a(t) v(t)+\sum_{i=1}^{m} b_{i}(t)[v(t)]_{\tau_{i}}, \\
v(t)=\phi(t), \quad t \in\left(-\infty, t_{0}\right] .
\end{array}\right.
$$

In references $[3,6-8,15,16,20,25,28,31,32,34-36]$, those results exploited the inequality (2.5) or (2.6) or (2.7). Therefore, those inequalities can be regarded as special cases of Theorem 2.1. That is to say, the conclusions of those above inequalities are a special case of our results.

Remark 2.3. In this paper, the proof method of Theorem 2.1 is different to those in reference $[3,8,32,36]$. Our methods is easy to understand and the proof is not complicated.

Remark 2.4. In reference [36], the authors considered the following system:

$$
v^{\prime}(t) \leqslant-a(t) v(t)+b(t) \sup _{t-\tau \leqslant s \leqslant t} v(s), \quad t \geqslant t_{0},
$$

they require $a(t) \geqslant a>0$ and $0<b(t) \leqslant q a(t)$ for all $t \geqslant t_{0}$ with $0 \leqslant q<1$. In fact, $a(t) \geqslant a>0$ and $0<b(t) \leqslant q a(t)$ is too limited. However, in Theorem 2.1, we only need $a(t)$ and $b(t)$ satisfy the following relationship

$$
-a(t)+b(t) \leqslant-\sigma<0,
$$

where $\sigma$ is a constant.

Remark 2.5. In the generalized Halanay's inequality of Theorem 2.1, the $\tau(t)$ can not a constant. In addition, we do not request that $t-\tau(t) \rightarrow+\infty$ when $t \rightarrow+\infty$. 


\section{Dissipativity and stability of delay integro-differential equations}

\subsection{Perturbed delay integro-differential equations by small $€$.}

Singularly perturbed delay differential equations arise in the study of an "optically bistable device" and in a variety of models for physiological processes or diseases. Such a problem has also appeared to describe the so-called human pupil-light reflex.

For example, Ikeda adopted the model

$$
\epsilon y^{\prime}(t, \epsilon)=-y(t, \epsilon)+A^{2}[1+2 B \cos (y(t-1, \epsilon))],
$$

to describe an optically bistable device and showed numerically that instability or chaotic behavior occurs for small $\epsilon$ and certain values of $A, B$, for the following system:

$$
\begin{cases}\epsilon y^{\prime}(t, \epsilon)=f\left(t, y(t, \epsilon), y(t-\tau(t), \epsilon), \int_{0}^{\sigma(t)} K(s) y((t-s), \epsilon) d s\right), & t \geqslant t_{0} \\ y(t, \epsilon)=\phi(s), & t \leqslant t_{0}\end{cases}
$$

and

$$
\begin{cases}\epsilon z^{\prime}(t, \epsilon)=f\left(t, z(t, \epsilon), z(t-\tau(t), \epsilon), \int_{0}^{\sigma(t)} K(s) z((t-s), \epsilon) d s\right), & t \geqslant t_{0} \\ z(t, \epsilon)=\psi(s), & t \leqslant t_{0} .\end{cases}
$$

Definition 3.1. The problem is said to be dissipative in $H$ if there exists a bounded set $B \in H$, such that for any given bounded set $\Phi \subset \mathrm{H}$, there is a time $\mathrm{t}^{*}=\mathrm{t}^{*}(\Phi)$, such that for any given initial function $\varphi \in \mathrm{C}[-\tau, 0]$ with $\varphi(t)$ contained in $\Phi$ for all $\mathrm{t} \in[-\tau, 0]$, the values of the corresponding solution $\mathrm{y}(\mathrm{t})$ of the problem are contained in $B$ for all $t \geqslant t^{*}$. Here $B$ is called an absorbing set of the problem.

Definition 3.2 (Exponentially stable uniformly for small $\epsilon$ ). The solution $y(t, \epsilon)$ of Eq. (3.1) is said to be exponentially stable uniformly for sufficiently small $\epsilon$ if it is asymptotically stable and there exist finite constants $K>0, \gamma>0$, and $\delta>0$, which are independent of $\epsilon \in\left(0, \epsilon_{0}\right]$ for some $\epsilon_{0}$ such that $\| y(t, \epsilon)-$ $z(t, \epsilon) \| \leqslant K e^{-\gamma\left(t-t_{0}\right)}$ for $t \geqslant t_{0}$ and for any initial perturbation satisfying $\sup _{s \in\left[t_{0}-\tau, t_{0}\right]}\|\phi(s)-\psi(s)\|<\delta$. Here $z(t, \epsilon)$ is the solution of Eq. (3.2) corresponding to the initial function $\psi$.

Theorem 3.3. Consider the system (3.1) and (3.2), where $f$ is sufficiently differential with respect to both the last three variables $0 \leqslant \tau(t) \leqslant \tau^{*}$, where $\tau^{*}$ is a constant, and the initial functions $\phi(\mathrm{s})$ and $\psi(\mathrm{s})$ are bounded and continuous for $-\infty<\mathrm{t} \leqslant \mathrm{t}_{0}$ and $\int_{0}^{+\infty} \mathrm{K}(\mathrm{s}) \mathrm{e}^{\mu \mathrm{s}} \mathrm{d} \mathrm{s}<+\infty(\mu>0)$. Suppose

$$
\begin{array}{r}
\operatorname{Re}\left\langle f\left(t, y_{1}, u, v\right)-f\left(t, y_{2}, u, v\right), y_{1}-y_{2}\right\rangle \leqslant \eta(t)\left\|y_{1}-y_{2}\right\|, t \geqslant 0, u, v, y_{1}, y_{2} \in \mathbb{C}^{s}, \\
\left\|f\left(t, y, u_{1}, v_{1}\right)-f\left(t, y, u_{2}, v_{1}\right)\right\| \leqslant \zeta(t)\left\|u_{1}-u_{2}\right\|+\theta(t)\left\|v_{1}-v_{2}\right\|, t \geqslant 0,
\end{array}
$$

where $\mathrm{y}, \mathrm{u}_{1}, \mathrm{u}_{2}, v_{1}, v_{2} \in \mathbb{C}^{\mathrm{s}}$, and $\eta(\mathrm{t})<0, \sigma>0, \zeta(\mathrm{t}), \theta(\mathrm{t})$ are continuous and satisfy

$$
\eta(t)+\zeta(t)+\theta(t) \int_{0}^{+\infty} K(s) d s \leqslant-\sigma<0, \quad t \geqslant t_{0}
$$

where $\mathbb{C}^{s}$ is the s-dimensional complex vector space, $\mathfrak{R}\langle\cdot, \cdot\rangle$ is real parts, and $\|\cdot\|$ is the induced norm of the inner product $\langle u, v\rangle=v^{\top} u$.

If (3.1) and (3.2) each has a unique solution, then there exists a small $\epsilon_{0}>0$ such that the solution of (3.1) is exponentially stable uniformly for sufficiently small $\epsilon \in\left(0, \epsilon_{0}\right]$.

Proof. According to the definition of the norm on $\mathbb{C}^{s}$, we have

$$
\frac{1}{2} \epsilon \frac{d}{d t}\left(\|y(t, \epsilon)-z(t, \epsilon)\|^{2}\right)=\operatorname{Re}\left\langle\epsilon y^{\prime}(t, \epsilon)-\epsilon z^{\prime}(t, \epsilon), y(t, \epsilon)-z(t, \epsilon)\right\rangle
$$




$$
\begin{aligned}
= & \operatorname{Re}\left\langle f\left(t, y(t, \epsilon), y(t-\tau(t), \epsilon), \int_{0}^{\sigma(t)} K(s) y((t-s), \epsilon) d s\right)\right. \\
& \left.-f\left(t, z(t, \epsilon), z(t-\tau(t), \epsilon), \int_{0}^{\sigma(t)} K(s) z((t-s), \epsilon) d s\right), y(t, \epsilon)-z(t, \epsilon)\right\rangle \\
= & \operatorname{Re}\left\langle f\left(t, y(t, \epsilon), y(t-\tau(t), \epsilon), \int_{0}^{\sigma(t)} K(s) y((t-s), \epsilon) d s\right)\right. \\
& \left.-f\left(t, z(t, \epsilon), y(t-\tau(t), \epsilon), \int_{0}^{\sigma(t)} K(s) y((t-s), \epsilon) d s\right), y(t, \epsilon)-z(t, \epsilon)\right\rangle \\
& +\operatorname{Re}\left\langle f\left(t, z(t, \epsilon), y(t-\tau(t), \epsilon), \int_{0}^{\sigma(t)} K(s) y((t-s), \epsilon) d s\right)\right. \\
& \left.-f\left(t, z(t, \epsilon), z(t-\tau(t), \epsilon), \int_{0}^{\sigma(t)} K(s) z((t-s), \epsilon) d s\right), y(t, \epsilon)-z(t, \epsilon)\right\rangle .
\end{aligned}
$$

Application of Schwartz'a inequality yields

$$
\begin{aligned}
\frac{1}{2} \epsilon & \frac{d}{d t}\left(\|y(t, \epsilon)-z(t, \epsilon)\|^{2}\right) \\
\leqslant & \left.\left.\eta(t)\|y(t, \epsilon)-z(t, \epsilon)\|^{2}+\zeta(t)\|y(t, \epsilon)-z(t, \epsilon)\| \| y(t-\tau(t)), \epsilon\right)-z(t-\tau(t)), \epsilon\right) \| \\
& +\theta(t)\|y(t, \epsilon)-z(t, \epsilon)\|\left\|\int_{0}^{\sigma(t)} K(s) y((t-s), \epsilon) d s-\int_{0}^{\sigma(t)} K(s) z((t-s), \epsilon) d s\right\| \\
\leqslant & \eta(t)\|y(t, \epsilon)-z(t, \epsilon)\|^{2}+\zeta(t)\|y(t, \epsilon)-z(t, \epsilon)\| \sup _{t-\tau(t) \leqslant s \leqslant t}\|y((s), \epsilon)-z((s), \epsilon)\| \\
& +\theta(t)\|y(t, \epsilon)-z(t, \epsilon)\|\left\|\int_{0}^{\sigma(t)} K(s)(y((t-s), \epsilon)-z((t-s), \epsilon)) d s\right\| \\
\leqslant & \eta(t)\|y(t, \epsilon)-z(t, \epsilon)\|^{2}+\zeta(t)\|y(t, \epsilon)-z(t, \epsilon)\| \sup _{t-\tau(t) \leqslant s \leqslant t}\|y((s), \epsilon)-z((s), \epsilon)\| \\
& \left.\left.+\theta(t) \int_{0}^{+\infty} K(s) d s\|y(t, \epsilon)-z(t, \epsilon)\| \sup _{-\infty<s \leqslant t} \| y(s), \epsilon\right)-z(s), \epsilon\right) \| \\
\leqslant & \eta(t)\|y(t, \epsilon)-z(t, \epsilon)\|^{2}+\zeta(t) \quad \sup _{t-\tau(t) \leqslant s \leqslant t}\|y((s), \epsilon)-z((s), \epsilon)\|^{2} \\
& \left.\left.+\theta(t) \int_{0}^{+\infty} K(s) d s \sup _{-\infty<s \leqslant t} \| y(s), \epsilon\right)-z(s), \epsilon\right) \|^{2} .
\end{aligned}
$$

Denote $V(t, \epsilon)=\|y(t, \epsilon)-z(t, \epsilon)\|^{2}$. It follows from (3.3) with $\epsilon>0$ that

$$
V^{\prime}(t, \epsilon) \leqslant \frac{2 \eta(t)}{\epsilon} V(t, \epsilon)+\frac{2 \zeta(t)}{\epsilon} \sup _{t-\tau(t) \leqslant s \leqslant t} V(s, \epsilon)+\frac{2 \theta(t)}{\epsilon} \int_{0}^{+\infty} K(s) d s \sup _{-\infty<s \leqslant t} V(s, \epsilon) .
$$

A direct application of Theorem 2.1 yields,

$$
\mathrm{V}(\mathrm{t}, \epsilon) \leqslant \tilde{\mathrm{G}} \mathrm{e}^{-\mu^{*}(\epsilon)\left(\mathrm{t}-\mathrm{t}_{0}\right)}, \quad \mathrm{t} \geqslant \mathrm{t}_{0} .
$$

Here

$$
\mu^{*}(\epsilon)=\inf _{t \geqslant t_{0}}\left\{\mu(t): \mu(t)+\frac{2 \eta(t)}{\epsilon}+\frac{2 \zeta(t)}{\epsilon} e^{\mu \tau(t)}+\frac{2 \theta(t)}{\epsilon} \int_{0}^{+\infty} K(s) e^{\mu s} d s=0\right\},
$$

and $\tilde{G} \geqslant 0$ only depends on the initial condition $\|\phi(t)-\psi(t)\|$.

For any fixed $t \geqslant t_{0}$, let $\mu(t, \epsilon)$ be defined as the unique positive zero of

$$
\mu(t)+\frac{2 \eta(t)}{\epsilon}+\frac{2 \zeta(t)}{\epsilon} e^{\mu \tau(t)}+\frac{2 \theta(t)}{\epsilon} \int_{0}^{+\infty} K(s) e^{\mu s} d s=0 .
$$


It can be proved that $\mu\left(t, \epsilon_{1}\right) \geqslant \mu\left(t, \epsilon_{2}\right)$ whenever $\epsilon_{2} \geqslant \epsilon_{1}>0$. This implies $\mu^{*}\left(\epsilon_{1}\right) \geqslant \mu^{*}\left(\epsilon_{2}\right)$ and thus we proved that $\mu^{*}(\epsilon)$ is monotonically decreasing with respect to the variable $\epsilon$. Hence we deduce that there exists a small $\epsilon_{0}$ such that the solution $y(t, \epsilon)$ is exponentially stable uniformly for sufficiently small $\epsilon \in\left(0, \epsilon_{0}\right]$. This completes the proof.

\subsection{Application to Volterra delay integro-differential equations}

Consider the initial value problem in Volterra delay integro-differential equations

$$
\left\{\begin{array}{lc}
x^{\prime}(t)=g\left(t, x(t), x(t-\eta(t)), \int_{0}^{\sigma(t)} K(t, s, x(t-s)) d s\right), & t \geqslant 0, \\
x(t)=\phi(t), & -\infty<t \leqslant 0,
\end{array}\right.
$$

where $\eta(t), \sigma(t) \in C[0,+\infty)$ with $-\tau \leqslant \eta(t) \leqslant t, \sigma(t)$ is a bounded function, the initial function $\phi(t)$ is bounded and continuous and the continuous mapping $K:[0,+\infty) \times[-\tau,+\infty) \times R \rightarrow R$ satisfying $\|\mathrm{K}(\mathrm{t}, \mathrm{s}, x(\mathrm{t}-\mathrm{s}))\| \leqslant \mathrm{k}(\mathrm{t}, \mathrm{s})\|x(\mathrm{t}-\mathrm{s})\|, \mathrm{k}(\mathrm{t}, \mathrm{s})>0$ and $\int_{0}^{+\infty} \mathrm{k}^{2}(\mathrm{t}, \mathrm{s}) \mathrm{ds}<+\infty$, and continuous mapping $\mathrm{g}:[0,+\infty) \times \mathrm{R} \times \mathrm{R} \times \mathrm{R} \rightarrow \mathrm{R}$ satisfying

$$
\frac{1}{2} \operatorname{Re}\left\langle u, g\left(t, u, v_{1}, v_{2}\right)\right\rangle \leqslant \gamma(t)+\alpha(t)\|u\|^{2}+\beta(t)\left\|v_{1}\right\|^{2}+\omega(t)\left\|v_{2}\right\|^{2},
$$

$\mathrm{t} \in[0,+\infty), \mathrm{u}, v_{1}, v_{2} \in \mathrm{R}$, where $\alpha(\mathrm{t})<0, \gamma(\mathrm{t}) \geqslant 0, \beta(\mathrm{t}) \geqslant 0, \omega(\mathrm{t}) \geqslant 0, \gamma^{*}=\sup _{0 \leqslant \mathrm{t}<+\infty} \gamma(\mathrm{t})$.

Thus the result of the Theorem 2.1 in the present paper can be directly applied to this special case, and we thus obtain the following dissipativity result.

Theorem 3.4. Suppose that $\mathrm{x}(\mathrm{t})$ is a solution of the problem (3.4) and there exists a constant $\sigma>0$ such that

$$
\alpha(t)+\beta(t)+\omega(t) \sigma(t) \int_{0}^{+\infty} k^{2}(t, s) d s \leqslant-\sigma<0, \quad t \geqslant 0 .
$$

Then

(i) for any given $\epsilon>0$, there exists a positive number $\mathrm{t}^{*}=\mathrm{t}^{*}\left(\|\varphi\|_{\infty}, \epsilon\right)$, such that

$$
\|y(t)\|^{2}<\frac{\gamma^{*}}{\sigma}+\epsilon, \quad t>t^{*}
$$

(ii) for any given $\epsilon>0$, the problem (3.4) is dissipative with an absorbing set $\mathrm{B}=\mathrm{B}\left(0, \sqrt{\frac{\gamma^{*}}{\sigma}+\epsilon}\right)$.

Proof. From $\|\mathrm{K}(\mathrm{t}, \mathrm{s}, \mathrm{x})\| \leqslant \mathrm{k}(\mathrm{t}, \mathrm{s})\|\mathrm{x}(\mathrm{t})\|$, we know,

$$
\begin{aligned}
\left\|\int_{0}^{\sigma(t)} K(t, s, x(t-s)) d s\right\| & \leqslant \int_{0}^{\sigma(t)}\|K(t, s, x(t-s))\| d s \\
& \leqslant \int_{0}^{\sigma(t)} k(t, s)\|x(t-s)\| d s<\int_{0}^{+\infty} k(t, s)\|x(t-s)\| d s .
\end{aligned}
$$

Let $V(t)=x^{2}(t)$, for $t \geqslant 0$,

$$
\begin{aligned}
\frac{d V}{d t}=2 x(t) x^{\prime}(t) & \leqslant \gamma(t)+\alpha(t)\|x\|^{2}+\beta(t)\|x(t-\eta(t))\|^{2}+\omega(t)\left\|\int_{0}^{\sigma(t)} K(t, s, x(t-s)) d s\right\| \\
& \leqslant \gamma(t)+\alpha(t)\|x\|^{2}+\beta(t) \sup _{-\eta(t) \leqslant s \leqslant t}\|x(s)\|^{2}+\omega(t) \sigma(t) \int_{0}^{+\infty} k^{2}(t, s)\|x(t-s)\|^{2} d s,
\end{aligned}
$$

by the proof of the Theorem 2.1, we can obtain the conclusions of the Theorem 3.4. 
3.3. Stability of multiple delay differential systems

$$
\left\{\begin{array}{lr}
x^{\prime}(t)=A x(t)+\sum_{i=1}^{n} B_{i} x\left(t-\tau_{i}(t)\right)+\sum_{j=1}^{m} C_{j} \int_{0}^{\sigma(t)} K_{j}(s) x(t-s) d s, & t \geqslant 0, \\
x(t)=\phi(t), & -\infty<t \leqslant 0,
\end{array}\right.
$$

where $x: \mathbb{R} \rightarrow \mathbb{R}^{n}$ is the state, $\tau_{\mathfrak{i}}(t) \geqslant 0,0 \leqslant \sigma(t) \leqslant \sigma^{*}$, the delay kernel $K_{j}(\cdot) \geqslant 0$ and $\int_{0}^{+\infty} K_{j}(s) e^{\mu s} d s<$ $+\infty(\mu>0), G=\sup _{-\infty<s \leqslant t_{0}}|\phi(s)|, A, B, C$ are $n \times n$ constant matrices.

In the following, we can conclude a sufficient condition for globally exponentially stable by the generalized Halanay inequality.

Theorem 3.5. The zero solution of (3.5) is globally exponentially stable if there exist $p<0, q_{i} \geqslant 0, w_{j} \geqslant 0$ and $n \times n$ matrices $P, Q_{i}, W_{j}>0(i=1,2, \ldots, n, j=1,2, \ldots, m)$, such that

$$
Q_{i} \leqslant q_{i} P, \quad W_{j} \leqslant w_{j} P, \quad p+\sum_{i=1}^{n} q_{i}+\sum_{j=1}^{m} w_{j} \sigma^{*} \int_{0}^{+\infty} K_{j}^{2}(s) d s<0,
$$

and

$$
M=\left(\begin{array}{rrrrrrr}
P A+A^{\top} P-p P & P_{1} & \cdots & P_{n} & P_{1} C_{1} & \cdots & P \\
B_{1}^{\top} P & -Q_{1} & \cdots & 0 & 0 & \cdots & 0 \\
\vdots & \vdots & \ddots & \vdots & \vdots & \vdots & \vdots \\
B_{n}^{\top} P & 0 & \cdots & -Q_{n} & 0 & \cdots & 0 \\
C_{1}^{\top} P & 0 & \cdots & 0 & -W_{1} & \cdots & 0 \\
\vdots & \vdots & \ddots & \vdots & \vdots & \ddots & \vdots \\
C_{m}^{\top} P & 0 & \cdots & 0 & 0 & \cdots & -W_{m}
\end{array}\right)_{(n+m+1) \times(n+m+1)} \leqslant 0
$$

hold.

Proof. Let $x$ be a solution of (3.5) and define $v(t)=x^{\top}(t) P x(t)$. Then

$$
\begin{aligned}
& v^{\prime}(t)=\left(x^{\prime}(t)\right)^{\top} P x(t)+x^{\top}(t) P x^{\prime}(t) \\
& =\left(A x(t)+\sum_{i=1}^{n} B_{i} x\left(t-\tau_{i}(t)\right)+\sum_{j=1}^{m} C_{j} \int_{0}^{\sigma(t)} K_{j}(s) x(t-s) d s\right)^{\top} P x(t) \\
& +x^{\top}(t) P\left(A x(t)+\sum_{i=1}^{n} B_{i} x\left(t-\tau_{i}(t)\right)+\sum_{j=1}^{m} C_{j} \int_{0}^{\sigma(t)} K_{j}(s) x(t-s) d s\right) \\
& =X\left(\begin{array}{rrrrrrr}
\mathrm{PA}+\mathrm{A}^{\mathrm{T} P} & \mathrm{~PB}_{1} & \cdots & \mathrm{PB}_{n} & \mathrm{PC}_{1} & \cdots & \mathrm{PC}_{\mathrm{m}} \\
\mathrm{B}_{1}^{\top} \mathrm{P} & 0 & \cdots & 0 & 0 & \cdots & 0 \\
\vdots & \vdots & \ddots & \vdots & \vdots & \vdots & \vdots \\
\mathrm{B}_{n}^{\top} \mathrm{P} & 0 & \cdots & 0 & 0 & \cdots & 0 \\
\mathrm{C}_{1}^{\top} \mathrm{P} & 0 & \cdots & 0 & 0 & \cdots & 0 \\
\vdots & \vdots & \ddots & \vdots & \vdots & \ddots & \vdots \\
\mathrm{C}_{\mathrm{m}}^{\mathrm{T}} \mathrm{P} & 0 & \cdots & 0 & 0 & \cdots & 0
\end{array}\right) \mathrm{X}^{\top} \\
& =X\left(\begin{array}{rrrrrrr}
\mathrm{PA}+\mathrm{A}^{\top} \mathrm{P}-\mathrm{pP} & \mathrm{PB}_{1} & \cdots & \mathrm{PB}_{\mathrm{n}} & \mathrm{PC}_{1} & \cdots & \mathrm{PC}_{\mathrm{m}} \\
\mathrm{B}_{1}^{\top} \mathrm{P} & -\mathrm{Q}_{1} & \cdots & 0 & 0 & \cdots & 0 \\
\vdots & \vdots & \ddots & \vdots & \vdots & \vdots & \vdots \\
\mathrm{B}_{n}^{\top} \mathrm{P} & 0 & \cdots & -\mathrm{Q}_{n} & 0 & \cdots & 0 \\
\mathrm{C}_{1}^{\top} \mathrm{P} & 0 & \cdots & 0 & -\mathrm{W}_{1} & \cdots & 0 \\
\vdots & \vdots & \ddots & \vdots & \vdots & \ddots & \vdots \\
\mathrm{C}_{\mathrm{m}}^{\top} \mathrm{P} & 0 & \cdots & 0 & 0 & \cdots & -W_{m}
\end{array}\right) X^{\top}
\end{aligned}
$$




$$
\begin{aligned}
& +p x^{\top}(t) P x(t)+\sum_{i=1}^{n} x^{\top}(t-\tau(t)) Q_{i} x(t-\tau(t)) \\
& +\sum_{j=1}^{m} \int_{0}^{\sigma(t)} K_{j}(s) x(t-s) d s W_{j} \int_{0}^{\sigma(t)} K_{j}(s) x(t-s) d s \\
& \leqslant p x^{\top}(t) P x(t)+\sum_{i=1}^{n} q_{i} x^{\top}\left(t-\tau_{i}(t)\right) P x\left(t-\tau_{i}(t)\right)+\sum_{j=1}^{m} w_{j} \sigma^{*} \int_{0}^{\sigma(t)} K_{j}^{2}(s) x^{\top}(t-s) P x(t-s) d s \\
& \leqslant p v(t)+\sum_{i=1}^{n} q_{i}[v(t)]_{\tau_{i}}+\sum_{j=1}^{m} w_{j} \sigma^{*} \int_{0}^{+\infty} K_{j}^{2}(s) v(t-s) d s,
\end{aligned}
$$

where

$$
X=\left(x(t), x\left(t-\tau_{1}(t)\right), \ldots, x\left(t-\tau_{n}(t)\right), \int_{0}^{\sigma(t)} K_{1}(s) x(t-s) d s, \ldots, \int_{0}^{\sigma(t)} K_{m}(s) x(t-s) d s\right) .
$$

Hence the conditions of Theorem 2.1 are satisfied, it yields

$$
v(\mathrm{t}) \leqslant \mathrm{Ge}^{-\mu^{*}\left(\mathrm{t}-\mathrm{t}_{0}\right)}, \quad \mathrm{t} \in \mathbb{R}^{+},
$$

where

$$
\mu^{*}=\inf _{t \geqslant t_{0}}\left\{\mu: \mu+p+\sum_{i=1}^{n} q_{i} e^{\mu \tau_{i}(t)}+\sum_{j=1}^{m} w_{j} \sigma^{*} \int_{0}^{+\infty} K_{j}^{2}(s) e^{\mu s} d s=0\right\} .
$$

Therefore, the zero solution of (3.5) is globally exponentially stable.

Remark 3.6. For the nonlinear system of Eq. (3.5), if given the Lipschitz condition or other conditions, it can also obtain some sufficient conditions for stability by the generalized Halanay inequality.

Remark 3.7. The inequalities (2.1) can be extended to the case of impulsive differential inequalities:

$$
\begin{cases}\mathrm{D}^{+} v(\mathrm{t}) \leqslant \gamma(\mathrm{t})+\mathrm{a}(\mathrm{t}) v(\mathrm{t})+\sum_{i=1}^{\mathrm{m}} \mathrm{b}_{\mathrm{i}}(\mathrm{t})[v(\mathrm{t})]_{\tau_{\mathrm{i}}}+\sum_{\mathrm{l}=1}^{\mathrm{n}} \mathrm{c}_{\mathrm{l}}(\mathrm{t}) \int_{0}^{+\infty} \mathrm{K}_{\mathrm{l}}(\mathrm{s}) v(\mathrm{t}-\mathrm{s}) \mathrm{ds}, & \mathrm{t} \neq \mathrm{t}_{\mathrm{k}}, \mathrm{t} \geqslant 0, \\ v\left(\mathrm{t}_{\mathrm{k}}\right) \leqslant \mathrm{p}_{\mathrm{k}} v\left(\mathrm{t}_{\mathrm{k}}^{-}\right)+\mathrm{q}_{1 k}\left[v\left(\mathrm{t}_{\mathrm{k}}^{-}\right)\right]_{\tau_{1}}+\mathrm{q}_{2 \mathrm{k}}\left[v\left(\mathrm{t}_{\mathrm{k}}^{-}\right)\right]_{\tau_{2}}+\cdots+\mathrm{q}_{\mathrm{mk}}\left[v\left(\mathrm{t}_{\mathrm{k}}^{-}\right)\right]_{\tau_{\mathrm{m}},} & \mathrm{t}=\mathrm{t}_{\mathrm{k}}, \mathrm{k} \in \mathbb{N}_{+}, \\ v(\mathrm{t})=\phi(\mathrm{t}), & \mathrm{t} \in\left(-\infty, \mathrm{t}_{0}\right] .\end{cases}
$$

In reference $[7,9-11,16,21,33-36]$, the conclusion of these inequalities can be used as a special case of the inequality (3.6).

\section{Application}

In this section, two examples are presented to demonstrate the effectiveness of our results about the dissipativity and stability of a class of nonlinear multiple time delay integro-differential equations.

Example 4.1. Consider the Volterra delay integro-differential equations:

$$
\left\{\begin{array}{lc}
x^{\prime}(t)=g\left(t, x(t), x(t-\eta(t)), \int_{0}^{\sigma(t)} K(t, s, x(t-s)) d s\right), & t \geqslant 0, \\
x(t)=\phi(t), & -\infty<t \leqslant 0 .
\end{array}\right.
$$

If

$$
\begin{aligned}
\frac{1}{2} \Re\left\langle x, g\left(t, x, x(t-\eta(t)), \int_{0}^{\sigma(t)} K(t, s, x(t-s)) d s\right)\right\rangle \leqslant & \gamma(t)+\alpha(t)\|x\|^{2} \\
& +\beta(t)\|x(t-\eta(t))\|^{2}+\omega(t)\left\|\int_{0}^{\sigma(t)} K(t, s, x(t-s)) d s\right\|^{2}
\end{aligned}
$$


where $G=\sup _{-\infty<s \leqslant t_{0}}|\phi(s)|,-1 \leqslant \eta(t)=\sin t \leqslant t, \sigma(t)=e^{2 t}, \gamma(t)=1+\frac{1}{1+t}, \gamma^{*}=2$

Let $k(t, s)=e^{-t-s}, \alpha(t)=-2 t-2, \beta(t)=t, \omega(t)=2 t$. It leads to

$$
\begin{aligned}
\alpha(t)+\beta(t)+\omega(t) \sigma(t) \int_{0}^{+\infty} k^{2}(t, s) d s & =-2 t-2+t+2 t e^{2 t} \int_{0}^{+\infty} e^{-2(t+s)} d s \\
& =-t-2+t=-2=-\sigma<0
\end{aligned}
$$

so, the conditions of Theorem 3.4 are satisfied.

From the Theorem 3.4, we obtained, for any given $\epsilon>0$, there exists a positive number $t^{*}=t^{*}(G, \epsilon)$, such that

$$
\|x(t)\|^{2}<1+\epsilon, \quad t>t^{*},
$$

and the problem (4.1) is dissipative with an absorbing set $B=B(0, \sqrt{1+\epsilon})$.

Example 4.2. Consider the stability of multiple delay differential systems:

$$
\left\{\begin{array}{lc}
x^{\prime}(t)=A x(t)+B x(t-\tau(t))+C \int_{0}^{\sigma(t)} K(s) x(t-s) d s, & t \geqslant 0, \\
x(t)=\phi(t), & -\infty<t \leqslant 0,
\end{array}\right.
$$

where $x=\left(x_{1}(t), x_{2}(t)\right)^{\top}, \tau(t)=2+\sin t, 0<\sigma(t)=5+\cos t<6=\sigma^{*}, G=\sup _{-\infty<s \leqslant t_{0}}|\phi(s)|$, the delay kernel $\mathrm{K}(\mathrm{t})=e^{-2 t}$, and $\mu=1$. If $\mathrm{A}=\left(\begin{array}{rr}-6 & 1 \\ 1 & -6\end{array}\right), \mathrm{B}=\left(\begin{array}{rr}1 & -0.5 \\ 0.4 & 1\end{array}\right), \mathrm{C}=\left(\begin{array}{rr}1 & -0.2 \\ 0.1 & 1\end{array}\right)$.

Let $p=-4, q=0.5, w=1$, then $p+q+w \sigma^{*} \int_{0}^{+\infty} K^{2}(s) d s=-2<0$. Through the MATLAB LMI toolbox calculation, we can obtain

$$
\mathrm{P}=\left(\begin{array}{ll}
0.4752 & 0.0927 \\
0.0927 & 0.4752
\end{array}\right), \quad \mathrm{Q}=\left(\begin{array}{ll}
0.1688 & 0.0312 \\
0.0312 & 0.1460
\end{array}\right), \quad \mathrm{W}=\left(\begin{array}{ll}
0.2742 & 0.0570 \\
0.0570 & 0.2661
\end{array}\right) .
$$

So, the conditions of Theorem 3.5 are satisfied. It follows from Theorem 3.5 that the zero solution of system (4.2) is globally exponentially stable.

Fig 1 . shows the effectiveness of the theory results we obtained.

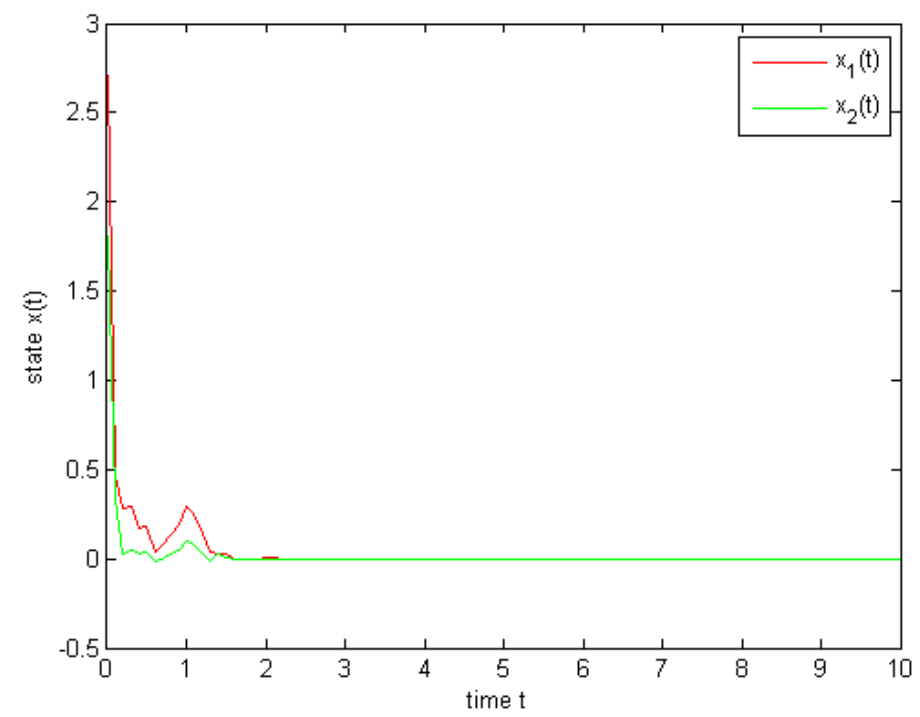

Figure 1: State trajectories of system (4.2). 


\section{Acknowledgment}

This work was supported by the National Natural Science Foundation of China under Grants 61573156, 61273126, 61503142, 61803095, 61803108, the Ph.D. Start-up Fund of Natural Science Foundation of Guangdong Province under Grant 2014A030310388, and the Fundamental Research Funds for the Central Universities under Grant x2zdD2153620, Science and Technology Plan Foundation of Guangzhou under Grant 201704030131.

\section{References}

[1] S. Arik, On the global dissipativity of dynamical neural networks with time delays, Phys. Lett. A, 326 (2004), 126-132. 1

[2] C. T. H. Baker, Development and application of Halanay-type theory: Evolutionary differential and difference equations with time lag, J. Comput. Appl. Math., 234 (2010), 2663-2682. 1

[3] C. T. H. Baker, A. Tang, Generalized Halanay inequalities for Volterra functional differential equations and discreted versions, in: Volterra Centennial Meeting, 2000 (2000), 39-55. 1, 2, 2.3

[4] Z. W. Cai, L. H. Huang, Functional differential inclusions and dynamic behaviors for memristor-based BAM neural networks with time-varying delays, Commun. Nonlinear Sci. Numer. Simul., 19 (2014), 1279-1300. 1

[5] M. El-Gebeily, D. O'Regan, Existence and boundary behavior for singular nonlinear differential equations with arbitrary boundary conditions, J. Math. Anal. Appl., 334 (2007), 140-156. 1

[6] A. Halanay, Differential Equations: Stability, Oscillations, Time Lags, Academic Press, New York-London, (1966). 1, 2

[7] D. W. C. Ho, J. L. Liang, J. Lam, Global exponential stability of impulsive high-order BAM neural networks with timevarying delay, Neural Networks, 19 (2006), 1581-1590. 1, 3.7

[8] C. X. Huang, Y. G. He, H. Wang, Mean square exponential stability of stochastic recurrent neural networks with timevarying delays, Comput. Math. Appl., 56 (2008), 1773-1778. 1, 2, 2.3

[9] X. D. Li, Existence and global exponential stability of periodic solution for impulsive CohenCGrossberg-type BAM neural networks with continuously distributed delays, Appl. Math. Comput., 215 (2009), 292-307. 3.7

[10] X. D. Li, M. Bohner, Exponential synchronization of chaotic neural networks with mixed delays and impulsive effects via output coupling with delay feedback, Math. Comput. Modelling, 52 (2010), 643-653.

[11] X. D. Li, M. Bohner, An impulsive delay differential inequality and applications, Comput. Math. Appl., 64 (2012), 1875-1881. 3.7

[12] B. Li, D. Xu, Mean square asymptotic behavior of stochastic neural networks with infinitely distributed delays, Neurocomputing, 72 (2009), 3311-3317. 1

[13] X. Liao, J. Wang, Global dissipativity of continuous-time recurrent neural networks with time delay, Phys. Rev. E, 68 (2003), 7 pages. 1

[14] X. Y. Liu, T. P. Chen, J. D. Cao, W. L. Lu, Dissipativity and quasi-synchronization for neural networks with discontinuous activations and parameter mismatches, Neural Networks, 24 (2011), 1013-1021. 1

[15] B. Liu, W. L. Lu, T. P. Chen, Generalized Halanay inequalities and their applications to neural networks with unbounded time-varying delays, IEEE Trans. Neural Networks, 22 (2011), 1508-1513. 1, 2

[16] X. Z. Liu, K. L. Teo, B. G. Xu, Exponential stability of impulsive high-order Hopfield-type neural networks with timevarying delay, IEEE Trans. Neural Networks, 16 (2005), 1329-1339. 1, 2, 3.7

[17] Y. Liu, Z. Wang, X. Liu, Global asymptotic stability of generalized bi-directional as sociative memory networks with discrete and distributed delays, Chaos Solitons Fractals, 28 (2006), 793-803. 1

[18] K. Y. Liu, H. Q. Zhang, An improved global exponential stability criterion for delayed neural networks, Nonlinear Anal. Real World Appl., 10 (2009), 2613-2619. 1

[19] X. Y. Lou, B. T. Cui, Global robust dissipativity for integro-differential systems modeling neural networks with delays, Chaos Solitons Fractals, 36 (2008), 469-478. 1

[20] S. Mohamad, K. Gopalsamy, Continuous and discrete Halanay-type inequalities, Bull. Austral. Math. Soc., 61 (2000), 371-385. 1, 2

[21] S. Mohamad, K. Gopalsamy, H. Akça, Exponential stability of artificial neural networks with distributed delays and large impulses, Nonlinear Anal. Real World Appl., 9 (2008), 872-888. 3.7

[22] D. O'Regan, M. El-Gebeily, Existence, upper and lower solutions and quasilinearization for singular differential equations, IMA J. Appl. Math., 73 (2008), 323-344. 1

[23] J.-L. Shao, T.-Z. Huang, X.-P. Wang, Further analysis on global robust exponential stability of neural networks with time-varying delays, Commun. Nonlinear Sci. Numer. Simul., 17 (2012), 1117-1124. 1

[24] J.-L. Shao, T.-Z. Huang, S. Zhou, Some improved criteria for global robust exponential stability of neural networks with time varying delays, Commun. Nonlinear Sci. Numer. Simul., 15 (2010), 3782-3794. 1

[25] H. J. Tian, The exponential asymptotic stability of singularly perturbed delay differential equations with a bounded lag, J. Math. Anal. Appl., 270 (2002), 143-149. 1, 2

[26] Z. W. Tu, J. G. Jian, K. Wang, Global exponential stability in Lagrange sense for recurrent neural networks with both time-varying delays and general activation functions via LMI approach, Nonlinear Anal. Real World Appl., 12 (2011), 2174-2182. 1 
[27] Z. W. Tu, L. W. Wang, Z. W. Zha, J. Jian, Global dissipativity of a class of BAM neural networks with time-varying and unbound delays, Commun. Nonlinear Sci. Numer. Simul., 18 (2013), 2562-2570. 1

[28] L. S. Wang, D. Xu, Global exponential stability of Hopfield reaction-diffusion neural networks with time-varying delays, Sci. China Ser. F, 46 (2003), 466-474. 1, 2

[29] L. S. Wang, L. Zhang, X. H. Ding, Global dissipativity of a class of BAM neural networks with both time-varying and continuously distributed delays, Neurocomputing, 152 (2015), 250-260. 1

[30] L. P. Wen, W. S. Wang, Y. X. Yu, Dissipativity and asymptotic stability of nonlinear neutral delay integro-differential equations, Nonlinear Anal., 72 (2010), 1746-1754.

[31] L. P. Wen, Y. X. Yu, W. S. Wang, Genernalized Halanay inequalities for disspativity of Volterra functional differential equations, J. Math. Anal. Appl., 347 (2008), 169-178. 1, 2

[32] D. Xu, Z. C. yang, Impulsive delay differential inequality and stability of neural networks, J. Math. Anal. Appl., 305 (2005), 107-120. 1, 2, 2.3

[33] X. S. Yang, Z. C. Yang, Synchronization of TS fuzzy complex dynamical networks with time-varying impulsive delays and stochastic effects, Fuzzy Sets and Systems, 235 (2014), 25-43. 3.7

[34] D. Yue, S. F. Xu, Y. Q. Liu, A differential inequality with delay and impulse and its applications to the design of robust controllers, Control Theory Appl., 16 (1999), 519-524. 1, 2

[35] Y. Zhang, J. T. Sun, Stability of impulsive functional differential equations, Nonlinear Anal., 68 (2008), $3665-3678$.

[36] Y. Zhang, J. T. Sun, Stability of impulsive linear hybrid systems with time delay, J. Syst. Sci. Complex., 23 (2010), 738-747. 1, 2, 2.3, 2.4, 3.7

[37] X. Y. Zhao, F. Q. Deng, Moment stability of nonlinear discrete stochastic systems with time-delays based on $H$ representation technique, Automatica J. IFAC, 50 (2014), 530-536. 1

[38] C. Zheng, N. L. JindeCao, Matrix measure based stability criteria for high-order neural networks with proportional delay, Neurocomputing, 149 (2015), 1149-1154. 1 